\title{
Introduction to the minitrack on Disaster Information, Technology, and Resilience in Digital Government
}

\author{
Frederick Benaben \\ IMT Mines Albi \\ frederick.benaben \\ @mines-albi.fr
}

\author{
Julie Dugdale \\ IMAG \\ Julie.Dugdale@im \\ ag.fr
}

\author{
Elsa Negre \\ Paris-Dauphine \\ elsa.negre@,dauphi \\ ne.fr
}

\author{
Mihoko Sakurai \\ Intl Univ. of Japan \\ msakurai@glocom \\ .ac.jp
}

\author{
Andrea Tapia \\ Penn State Uni. \\ atapia@ist.psu.edu
}

Clearly, the year 2020 has shown how sensitive human society is to crisis situations. This is not only a simple matter of fact, this is also and mainly the consequence of the hyper connection of this human society. There is actually no more absorption space for our world and society to absorb any significant disruption and avoid its spreading across the whole planet.

However, the progress of human society, in addition to being considered as the main cause of this dangerous evolution, could also provide avenues to deal with it: data science, artificial intelligence, social networks and more globally information technologies are amazing avenues that "in charge" institutions and governments should deeply consider to take the right path to deal with the tremendous next challenges of the century (global warming, pandemics, overpopulation and lack of resources, disbalance north and south, drop of biodiversity, etc.).

The DIRECT minitrack of the eGov track of HICSS 54 presents nine great papers addressing these issues but also these perspectives. The following presents each of these articles.

\section{Designing Chemical Emergency Response Systems Based on Open Data Tobias Enders, Niklas Kühl, Jannis Walk, Marc Muff}

This article addresses shortcomings in leveraging information technology in emergency situations that involve chemical substances. The authors integrate data provided by private organisations as Open Data with emergency services. This article proposes three contributions, as outcomes of the adopted Design Science Research methodology: 1. Identifying five design requirements for an artefact for emergency scenarios 2. Design and evaluation of such an artefact to assess its suitability 3 . Assessing the artefact and Open Data contributions.

\section{Crisis management and distrust: Study of an industrial accident in France Elsa Negre}

Elsa Negre, in her article "Crisis management and distrust: Study of an industrial accident in France" looks at the distrust that people may have towards public authorities and their decisions. The author describes how the mistrust of populations towards public policies can be explained by the fact that: (i) citizens are more and more qualified and therefore more and more capable of taking an interest in debates and giving their opinion, (ii) numerous scandals have tarnished the confidence of populations (contaminated blood, Chernobyl, etc.), (iii) France's growth is slowing down, which raises doubts about the effectiveness of public authorities' decisions, (iv) digital and social networks weaken the credibility of experts (political, scientific, etc.). The answers from an online questionnaire concerning the industrial accident at the Lubrizol factory in Rouen, France, in 2019 gives some practical leads on how an information system could reconcile populations and decision-makers.

\section{Citizens' behaviours related to smoke in bushfires and their implications for computational models of evacuation Julie Dugdale, Vincent Lemiale}

The behaviour of citizens strongly influences the degree to which they will be affected by a disaster, whether it is the Covid-19 pandemic or bushfires, as addressed in the paper by Julie Dugdale and Vincent Lemiale. In their paper "Citizens' behaviours related to smoke in bushfires and their implications for computational models of evacuation", the authors show how smoke is an important factor that can trigger peoples behaviours. The authors uncover 5 common behaviours exhibited by people in response to smoke from a serious bushfire. Effective evacuation from a bushfire is a critical issue, which has been aided by 
computer simulations that can assess different strategies and policies. It is now recognised that human behaviours must be taken into account in such simulations, but how people react to bushfire smoke is rarely included. In order to develop realistic simulators that can help to plan evacuations, the authors argue that smoke related behaviours should be included.

\section{Open Science at a time of the COVID-19 pandemic: a new opportunity to improve emergency response}

Hanna Shmagun, Charles Oppenheim, Jangsup Shim, Kwang-Nam Choi, Jaesoo Kim

In the paper "Open Science at a time of the COVID-19 pandemic: a new opportunity to improve emergency response", Hanna Shmagun and her colleagues identify diverse factors influencing attitudes to OS data sharing and reuse practices in public health emergencies, such as the current COVID-19 pandemic. The work identifies 4 primary categories' (contextual or external, institutional and regulatory, resource, individual and motivational) within which they discuss multiple factors. From the paper, it is clear that a national scholarly communication system based on OS principles cannot be built overnight in sudden crisis situations, but that multiple processes, including normative structures and basic infrastructure, should be systematically prepared before a crisis hits.

\section{Characterizing 311 System Reactions to a Global Health Emergency Duygu Pamukcu, Christopher W. Zobel}

Duygu Pamukcu and Christopher W. Zobel, in their paper "Characterizing 311 System Reactions to a Global Health Emergency" looks at how Citizen Relationship Management systems can be used to gather specific information from citizens complaints to collect and analyze information during an emergency. 311 Call Systems are non-emergency municipal service lines destinated for non-emergency local government service requests. 311 calls can be used as indicators of citizen reactions to a crisis situation. Indeed, it is clear from the paper that using data provided by 311 Call Systems to explore citizens' reactions to COVID-19 pandemic can generate rich discussions and increased understanding of people's behavior during a crisis.

\section{Social media processing in crisis response: an attempt to shift from data to information exploitation}

Julien Coche, Guillermo Romera Rodriguez, Aurélie Montarnal, Andrea Tapia, Frederick Benaben

Data coming from social media has helped to inform decision-makers on the best actions to take. However, this requires significant human input. In the paper, "Social media processing in crisis response: an attempt to shift from data to information exploitation" Julien Coche and his colleagues investigate a way to automatically organize information from social media data up to decision-makers. By unravelling how social media processing for decision makers usually works, the authors then present a meta-model that allows representing and using information so that different actors can collaborate during the crisis response. Pushing their work further, they then show how the classes in the meta-model may be implemented.

\section{Building Community Resilience Using Gain-Loss Framing to Nudge Homeowner Mitigation and Insurance Decision-making Mengtian Zhao, Richard John}

In disaster management, it is critical to understand how individuals make decisions when facing natural disasters so as to better allocate resources and help develop effective communication strategies. In the paper by Mengtian Zhao and Richard S. John on "Building Community Resilience Using Gain-Loss Framing to Nudge Homeowner Mitigation and Insurance Decision-making" the authors investigate whether manipulating the decision frame for taking protective action influences the likelihood of adopting mitigation measures. A behavioral experiment was used to test whether gain-loss framing can be used to nudge homeowner risk mitigation and insurance purchase decisions. The results were positive and furthermore showed that the approach is generalisable across several natural disasters.

\section{Machine Learning for Readability Assessment and Text Simplification in Crisis Communication: A systematic Review}

Hieronymus Hansen, Adam Widera, Johannes Ponge, Bernd Hellingrath

In the paper "Machine Learning for Readability Assessment and Text Simplification in Crisis Communication: A systematic Review", Hieronymus Hansen, Adam Widera, Johannes Ponge and Bernd Hellingrath explore the use of machine learning for the generation of messages intended for populations in crisis situations and thus tend towards successful Crisis 
Communication. According to the authors, readability assessment has the potential for an effective use in crisis communication, but there is a lack of sufficient training data. To overcome this lack, text simplification is used, where an exact assessment is only partly possible.

\section{Data Census of a Geographically- Bounded Tweet Set to Enhance Common Operational Picture Tools}

Nathan J. Elrod, Howard Hall, Pranav Mahajan, Rob Grace and Jess Kropczynski

Nathan J. Elrod, Howard Hall, Pranav Mahajan, Rob Grace and Jess Kropczynski, in "Data Census of a Geographically-Bounded Tweet Set to Enhance Common Operational Picture Tools" focus on location information which is of great importance in a crisis situation. This paper provides a comparison of location information provided by Twitter's four geolocation methods. Using real data, the authors evaluate the relative contribution that each method can make to common operational picture tools used by crisis informatics researchers. The study reveals that location information available for collection in hyperlocal geographic area is mostly course-grained, involving city and place names entered or tagged by users, respectively. These hyperlocal observations compliment national and global analyzes of geotagging behavior in previous benchmarked studies and fill in gaps on geographic information behavior in crisis informatics literature. 